\title{
Bienestar y salud: un marco para el análisis comparativo sobre el origen y desarrollo de los Sistemas de Salud
}

\section{Welfare and health: a framework for comparative analysis on the origin and development of Health Systems}

\author{
Marcelo Setaro Montes de Oca ${ }^{a}$
}

\begin{abstract}
Resumen
Este artículo presenta un marco analítico para el estudio comparativo sobre el origen y desarrollo de uno de los componentes centrales de los Estados de Bienestar: los sistemas de servicios de salud. El trabajo procura facilitar la comprensión de los procesos de construcción de sistemas de salud con orientación universalista, identificando los principales hitos en su construcción, así como los desafíos que se plantean para su estabilidad y desarrollo. El enfoque se construye a partir del análisis de la experiencia de los sistemas de protección en salud maduros de países desarrollados, procurando aportar pistas a los países latinoamericanos que buscan avanzar en la senda de implantar sistemas de salud universales. Construir sistemas de salud universales es una tarea fundamental en la afirmación del estatuto de ciudadanía, componente esencial para una democracia sustantiva.
\end{abstract}

Palabras clave: estado de bienestar, políticas públicas, salud.

\begin{abstract}
This article presents an analytical framework for the comparative study on the origin and development of one of the central components of Welfare State: the Health System. The work seeks to facilitate the understanding of the processes of construction of health systems with a universalistic orientation, identifying the milestones in its construction, as well as the challenges that are posed for its stability and development. The approach is built on the analysis of the experience of mature health protection systems in developed countries, seeking to provide clues to Latin American countries that seek to advance the path of implementing universal health systems. Building universal health systems is a fundamental task in affirming the statute of citizenship, an essential component of a substantive democracy.
\end{abstract}

Keywords: welfare state, public policies, health.
Kera Yvoty: reflexiones sobre la cuestión social. Vol. 2, 2017,

ISSN' (impreso): 2519-7797

a Universidad de la República, Facultad de Ciencias Sociales, Uruguay.

Correspondencia a: marcelosetaro@gmail.com

Cita:

Setaro de Oca, M. (2017).

Bienestar y Salud: un marco

para el análisis comparativo sobre el origen y desarrollo de los Sistemas de Salud. Kera Yvoty: reflexiones sobre la cuestión social, 2, 57-71.

Recibido:

30 agosto 2017

Aceptado:

29 noviembre 2017 


\section{Introducción}

Este artículo presenta un marco analítico para el estudio comparativo sobre el origen y desarrollo de los modernos sistemas de servicios de salud. Este marco procura facilitar la comprensión de los procesos de construcción de sistemas de salud con orientación universalistas, identificando los principales hitos en su construcción, así como los desafíos que se plantean para su estabilidad y desarrollo. Enfoque se construye a partir del análisis de la experiencia de los sistemas de protección en salud de los países desarrollados.

Para los países latinoamericanos resulta fundamental comprender los problemas que deben enfrentar frente a la implantación y el desarrollo de un sistema de atención en salud de vocación universal. Si se pretende avanzar en esta senda de democratización fundamental de las sociedades, la afirmación del estatuto de ciudadanía y los derechos sociales que forman parte de él, son un componente esencial para un estado de derecho democrático.

Los sistemas de servicios de salud son un componente crítico dentro de los modernos Estados de Bienestar. El nivel de los recursos económicos que se destinan al financiamiento de la atención médica, la importancia en cuanto a cantidad y formación de los recursos humanos involucrados, los recursos tecnológicos, todos estos elementos definen una compleja arena de política pública.

Este marcoanalítico debe permitirnos conocer las bases de funcionamiento de los sistemas de servicios de salud. Esto es, el proceso histórico de organización de las infraestructuras y tecnologías sanitarias, los recursos humanos, mecanismos de financiamiento, entre otros. Conocer el delicado funcionamiento de un sistema de salud, y cómo éstos gestionan las enormes demandas sociales que genera el aumento de la expectativa de vida y las presiones de la industria médica.
La primera parte aborda la conceptualización de la salud, como tema de política, y el enfoque analítico sobre sistemas de salud. Luego, la segunda parte trata sobre la génesis y evolución de los dos principales modelos de sistemas de salud, denominados en la literatura como Seguros Sociales de Salud (Social Health Insurance, SHI) y los Servicios Nacionales de Salud (National Health Services, NHS). Finalmente, las conclusiones establecen algunas recomendaciones de política, orientadas principalmente hacia los países de baja cobertura en salud de América Latina.

\section{Visiones sobre el concepto de salud}

En el año 1946, la Organización Mundial de la Salud (OMS) estableció que la Salud es el estado de completo bienestar físico, mental y social, y no solamente la ausencia de enfermedad. Esta definición recibió críticas por su excesiva ambición totalizadora y por lo poco operativa que resultaba. Pero es evidente que en su época supuso un gran avance, al definir a la salud de una manera positiva, y no solamente en términos de "ausencia de enfermedad". A las visiones más tradicionales, que limitaban el análisis de la salud como un fenómeno físico-orgánico ${ }^{1}$, agregó dos componentes adicionales, el bienestar mental y social de los individuos.

Esta mirada amplia sería compatible con otros desarrollos, de cuño sociológico y salubristas, que se enfocan sobre los fenómenos de causación social de la enfermedad. En ellos el bienestar social no es solamente un componente de la salud de las personas, sino también un medio para su consecución. La sociedad determina

\footnotetext{
1 Esta visión restringida se ha mantenido vigente en el tiempo. Por ejemplo, según el diccionario de la Real Academia Española, la salud es definida como "el estado en que el ser orgánico ejerce normalmente todas sus funciones" (RAE, 2017).
} 
la salud de las personas, al exponerlas a determinados riesgos ambientales que impactan en sus niveles de salud, y determinando las posibilidades de acceso a los recursos necesarios para gestionar esos riesgos. La pobreza incrementa la chance de estar expuesto a diversos riesgos: mala calidad de vivienda, polución ambiental, lugares de trabajo inseguros, etc. Además, el nivel de ingreso determina las posibilidades de contar con recursos necesarios para enfrentar esos riesgos, limitando el acceso a una alimentación saludable o a servicios de salud adecuados (Siegrist, 200o).

Esta visión sobre la determinación social de la salud tiene una larga tradición dentro de la medicina occidental. Los trabajos realizados en la segunda mitad del siglo XIX, por parte de John Snow sobre las epidemias de cólera en Inglaterra, y Rudolp Virchow sobre la fiebre tifoidea, identifican este vínculo entre condiciones de vida y salud. El acceso a agua potable, control de excretas, vivienda adecuada y alimentación saludable, son factores explicativos de la salud de las personas. Se identifica por primera vez que la propia organización de la sociedad (industrial) es un determinante fundamental de las posibilidades vitales de las personas. Según Virchow, la medicina, como ciencia social, debe incorporar dentro de sus objetivos la promoción de las reformas sociales radicales, la democracia, la educación, la libertad y la prosperidad (Navarro, 1997).

En realidad, estos desarrollos ya se venían procesando desde el siglo XVIII, con la consolidación de los Estados Nacionales europeos y el accionar del Despotismo Ilustrado. La Higiene Pública emerge como una disciplina científica, y como práctica de intervención del Estado para mejorar el bienestar de la población. En el nacimiento de esta nueva disciplina resulta determinante la obra científica de Johann Peter Frank (1779) en Alemania, quien establece la relación que existe entre pobreza y enfermedad, y sienta las bases de una ciencia dirigida a erradicar las bases de la enfermedad (Marset Campos et al., 2000).

Pero, con el impacto de los descubrimientos en el campo de la Microbiología, a partir de los descubrimientos de Luis Pasteur (186064) y Robert Koch (1884), la atención en salud comienza a generar un giro profundo hacia un modelo biomédico. La salud y enfermedad son vistos cada vez más como problemas individuales en una maquinaria biológica que puede ser reparada de manera fragmentaria, transfiriendo todo el protagonismo a las élites profesionales y la tecnología médica. La ciencia médica adquiere progresivamente, más y mejores instrumentos para el tratamiento de la enfermedad.Losenfoquessobrelacausación social de la enfermedad comienzan a pasar a un segundo plano. El milagro de la recuperación de la salud comienza a concentrarse en esa concentración del conocimiento tecnológico médico que es el moderno hospital (Setaro, 2013).

Habría que esperar a la publicación del célebre Reporte Lalonde de 1974, sobre la salud de los canadienses, el cual establece que el "campo de la salud" está determinado por una serie de factores. En primer lugar, los factores Sociales y Medioambientales, tales como la desigualdad socio-económica, hábitat, educación, etc. En segundo término, los Estilos de Vida, tales como el sedentarismo, alimentación, conductas de riesgo, entre otros. Luego, los Factores Biológicos de cada individuo, la carga genética de cada persona, que interactúa con los otros tres factores. El cuarto factor refiere a la organización de los serviciosdesalud, talescomoelfinanciamiento, la provisión, generación de insumos, sistemas de información y conocimiento, rectoría

El Reporte Lalonde establece que los sistemas de servicios de salud, en comparación con los otros tres factores mencionados, es quien realiza la menor contribución relativa al estado de salud de las personas. El excesivo enfoque biomédico sobre la salud constituye un error mayúsculo, porque no incide en los 
otros tres factores determinantes de la salud de las personas. El punto clave es el rol que puede llegar a jugar el sistema de salud en relación a los otros factores de mayor peso. Esto es, los equipos de salud pueden asumir un rol protagónico en la gestión de los riesgos sociales y medioambientales, y en la promoción de estilos de vida saludables en las personas. También es relevante su aporte, en términos de modelar la forma en que las personas gestionan su carga genética y riesgos de salud.

La OMS recogería esta visión más amplia, respecto a la interacción existente entre la salud de los individuos, sus condiciones de vida y el rol que podrían llegar a desempeñar los equipos de salud. Para este organismo, en el contexto de la promoción de la salud, la salud no debe ser considerada como un estado abstracto sino como un medio para llegar a un fin, como un recurso que permite a las personas llevar una vida individual, social y económicamente productiva. La salud es un recurso para la vida diaria, no el objetivo de la vida. Se trata de un concepto positivo que acentúa los recursos sociales y personales, así como las aptitudes físicas para desarrollar una vida satisfactoria. La promoción de la salud, en cuanto política pública, consiste en el proceso de fortalecer el control que las personas tienen sobre los factores que determinan su nivel de salud (Setaro, 2013).

El personal de salud y su organización es una variable clave en la explicación del funcionamiento del sector salud. Según Freidson (1970), el punto capital en la definición del proceso de salud y enfermedad tiene que ver con la organización social de la profesión médica. La medicina, como profesión, ha logrado una jurisdicción prácticamente exclusiva para determinar qué cosa es enfermedad y consecuentemente como se debe actuar frente a eso. El trabajo de la medicina consiste, precisamente, en realizar el diagnóstico y tratamiento degente quealega estar enferma. En ese sentido, la medicina tiene la autoridad para establecer si la queja de una persona es una enfermedad, y en ese proceso define las posibilidades sociales para representar la enfermedad.

En tanto profesión, se concede a la Medicina el poder oficial para definir los espacios problemáticos de la conducta social. El juez determina lo que es legal y quién es culpable, el clérigo lo que es sagrado y quién es profano, el médico los que es normal y quién está enfermo. En el proceso histórico de construcción de la autonomía y especificidad del campo profesional, los médicos se asocian con el Estado, que es el responsable de conceder el monopolio, pero también de autorizarlo, de regularlo.

Finalmente, el conflicto político ha sido un potente motor al proceso de construcción del sector salud. La política de salud se constituye en una arena de derechos sociales, en donde las prestaciones diversas como el aborto, la fertilización asistida, la psicoterapia, la atención odontológica, pugnan por ser considerados derechos que deben ser financiados por la comunidad. En ese proceso, la profesión médica y la sociedad organizada impulsan las fronteras de la intervención estatal, generando un proceso de estructuración, de institucionalización de este campo de la política pública.

\section{La estructura de los sistemas de salud}

Para tener una discusión racional es fundamental definir los límites y alcances de un sistema de salud. En un extremo del campo conceptual, podemos limitarlo a la atención médica de pacientes, con servicios organizados para tratar la enfermedad, discapacidad o la muerte. Pero en otro extremo, tenemos definiciones que abarcan todos aquellos determinantes que contribuyen de forma directa o indirecta en la salud de las personas. Es necesario un balance entre los dos extremos, entre la visión acotada sobre los servicios curativos, y la visión amplia sobre todo aquello que impacta en el bienestar de las personas (Figueras et al., 2010). 
Según la caracterización pionera de Roemer (1985), los sistemas de salud pueden ser analizados según cinco aspectos fundamentales: recursos, organización, funcionamiento, financiamiento y prestación de servicios. Villar (2003), va un poco más lejos y dice que un sistema de salud es un todo unitario y complejo, en el que sus componentes mantienen una relación ordenada, contribuyendo a un objetivo determinado, el de brindar servicios de salud para la población. Con base en definiciones de este tipo es que se podido impulsar un importante desarrollo en el campo de los estudios comparativos de los sistemas de salud ${ }^{2}$.

En la visión de Roemer (1985), los recursos consisten en la proporción de personal, infraestructura hospitalaria, insumos médicos y equipamientos, y conocimientos disponibles. La organización, en cuanto a la participación del Estado en la provisión, organizaciones benéficas y voluntarias, actores mercantiles. Elfuncionamiento, implica aspectos comola planificación, administración y legislación, responsabilidades de las profesiones, el papel de las acciones preventivas y las curativas. La financiación, refiere a las diferentes fuentes, sean estas públicas o privadas. La prestación de servicios en los diferentes niveles: el primario, en la prevención y la curación; el secundario y terciario en la atención especializada; la atención específica de determinados colectivos (según enfermedad, riesgo, edad, etc.).

Recientemente, la OMS nos propone una definición más simple de sistema de salud, el cual estaría compuesto por todas las organizaciones, personas e instituciones que producen acciones cuyo objetivo principal consiste en promover, restaurar o mantenerlasalud.Estadefinicióncomprende

2 En Whiteford y Lacivita (2000) hay un excelente resumen de la historia del campo y de los avances más recientes en términos de estudios comparativos sobre sistemas de salud. además aquellas acciones intersectoriales, que el Rector del sistema de salud asume como su responsabilidad de impulsar, en áreas ajenas a su dominio directo. Por ejemplo, el impulso a la legislación dirigida a combatir los accidentes de tránsito, o los impactos medioambientales sobre la salud de los emprendimientos productivos (Setaro, 2013).

La visión analítica de OMS permite superar definiciones normativas, estableciendo que todos los países tienen algún tipo de sistema de salud. La organización sanitaria de un país puede ser fragmentada, tener problemas de coordinación importantes, y que esto impacte negativamente en su desempeño, en su capacidad de lograr resultados de salud. Pero eso no obsta que estemos frente a un tipo de organización de los recursos de salud, y que podamos valorar la eficacia y eficiencia de esos arreglos.

Para Mladovsky et al. (2012), el grado de cumplimiento de los objetivos de los sistemas de salud es un indicador clave de la bondad de los mismos. Estos investigadores toman los objetivos generales de los sistemas de salud propuestos por OMS, pero los amplían a ocho puntos, que son la referencia para evaluar el funcionamiento de un buen sistema. Estos son:

i. Nivel de salud, mejoras de los resultados de salud poblacional y de los resultados de los servicios de salud

ii. Protección financiera, asegurando que la población no sufra cargas excesivas cuando requiera de atención en salud

iii. Eficiencia, tratando de maximizar las ganancias en salud poblacional, a partir de los niveles de inversión en salud, asegurando que los beneficios superen los costos. Las ganancias de eficiencia implican mantener los resultados de salud con un menor costo, mejores resultados de salud a un costo similar o mejores resultados 
con costos mayores, pero donde los beneficios exceden a los costos extra.

iv. Equidad, buscando que los servicios seasignen en función de la necesidad de atención y que las contribuciones al financiamiento sean acordes con la capacidad económica de las personas

v. Calidad, que combine efectividad clínica y satisfacción del paciente, considerando las dimensiones de seguridad, efectividad, accesibilidad y aceptabilidad.

vi. Sensibilidad a las demandas de los usuarios (Responsiveness), en términos de un tratamiento digno y sensible de los servicios de salud frente a sus necesidades.

vii. Transparencia, que permita apreciar los atributos del sistema de salud, tales como sus beneficios, costos y calidad.

viii. Responsabilización, que permita asociar el desempeño del sistema con las consecuencias esperadas de una acción correcta o incorrecta (una recompensa o un castigo).

Para poder cumplir con sus objetivos básicos, los sistemas de salud deben ejercery cumplir cuatro funciones básicas: Provisión de Servicios personales y poblacionales de salud, Financiamiento del sistema, Generación de Recursos e insumos básicos, ejercer la Rectoría de todo el sistema.

Los servicios que provee el sistema de salud pueden ser clasificados de acuerdo a su participación en el proceso de saludenfermedad (prevención, curación o rehabilitación) o por las tecnologías de que hace uso (laboratorio, radiología, etc.). Según los profesionales que participan en su provisión (médico, enfermera, cirujano, otros), o por los destinatarios de la atención (madre, niños, etc.). También, de forma más general, entre servicios de salud de orientación personal o poblacional3.

La segunda función, consiste en el Financiamiento del sistema e implica recolectar los recursos económicos de la sociedad, mancomunar los fondos y riesgos (pooling), y realizar la compra de servicios a los proveedores. Todos los sistemas de salud financian de alguna forma la provisión de servicios personales y poblacionales de salud, con el objetivo de conservar, mejorar o recuperar la salud de las personas amparadas. Las diversas formas de organizar este vínculo, de financiamiento y provisión, generalmente define diferentes tipos de sistemas de salud, como por ejemplo Servicios Nacionales o Seguros Sociales.

La tercera función del sistema refiere a la identificación, creación o desarrollo de los Recursos necesarios para producir servicios de salud y estructurar un sistema: conocimiento, recursos humanos, dispositivos tecnológicos, infraestructuras. Dada la naturaleza de los servicios de salud, intensiva en cuanto al uso de recursos humanos, la determinación del volumen, calificación y la forma de conformar los equipos de trabajo (skillmix) es fundamental. Además, los servicios requieren de modernos equipamientos y tecnologías, tanto para la atención de personas como de grupos poblacionales.

Finalmente, en cuarto lugar, la función de Rectoría consiste en el ensamble de las diferentes acciones estén orientadas al logro de los objetivos del sistema de salud. La Rectoría no solo incide sobre la ejecución de las otras funciones, sino que hace posible el logro de cada uno de los objetivos del sistema: mejorar la salud, responder a las legítimas expectativas de la población y una contribución justa al

\footnotetext{
3 Por ejemplo, los servicios personales reconocemos la atención quirúrgica, consulta médica, asesoría en salud, inmunización de un niño. Los servicios poblacionales son brindados a grupos más amplios, incluyen las campañas de inmunización, las advertencias en paquetes de cigarrillos, la promoción de salud en lugares de trabajo.
} 
financiamiento del sistema. Esto requiere de Ministerios de Salud con visión, inteligencia y capacidad de influencia, que actúan en nombre el gobierno nacional y en defensa de los intereses colectivos de la sociedad, formulando la política de salud, estableciendo la visión y la dirección del sistema y generando información en salud. También ejercen influencia, a través de la acción regulatoria.

Estas funciones de los sistemas de salud se construyen de forma histórica. La función Rectora, central en el desempeño del sistema de salud, refleja las capacidades del Estado para aplicar el conocimiento en las políticas de salud. Según el Informe Mundial de la Salud, en un estudio sobre 115 países de renta media y baja, para el período 1960-1990, la aplicación sistemática de conocimiento científico-técnico fue responsable de la reducción de casi un 50\% de la mortalidad 4 (OMS, 2010).

Pero para llegar a esta situación, los mecanismos colectivos de respuesta a las necesidades en salud debieron realizar un largo camino. Las instituciones sanitarias debieron evolucionar históricamente, para llegar a ser lo que conocemos hoy.

\section{Génesis histórica de los sistemas de salud}

La actividad de sanación frente a la dolencia y la invalidez ha existido desde la antigüedad. En el origen, la práctica médica se plasmaba como una relación directa, sin intermediación, con el paciente. El sanador o médico brindaba asistencia y cuidados, y los pacientes pagaban de manera directa por sus servicios. En esa relación, el médico muchas veces moderaba sus pretensiones económicas, en función de la capacidad económica del paciente. De esta forma, el médico era independiente respecto a la determinación de sus ingresos y las condiciones de su práctica (Starr, 1991)

4 En particular, la aplicación sistemática de programas de inmunización y el desarrollo y aplicación de antibióticos
El aumento de la complejidad en la asistencia médica, se refleja en el surgimiento y desarrollo de los modernos Hospitales. La concentración de medios tecnológicos en los servicios hospitalarios, de diversas profesiones con conocimientos especializados en el cuidado y la atención de la enfermedad. Pero habría que esperar hasta el siglo XX para que estos establecimientos se convirtieran en un enorme dispositivo organizativo, humano y tecnológico, dirigido a recuperar la salud.

El trabajo de Healy et al. (2002) nos muestra el largo periplo en la evolución de los Hospitales, que surgen como lugares vinculados a órdenes religiosas, durante la edad media, para la recepción y el cuidado de indigentes. Luego, en el siglo XVIII, pasan a ser un lugar en donde la profesión médica comienza a desarrollar y acumular conocimientos, y los pobres van a morir de una manera discreta. Finalmente, recién en el siglo XX se convierte en el símbolo central y brillante de la modernidad de los sistemas de salud.

Los ejemplos más antiguos que se reconocen como Hospitales se encuentran en el siglo VII en Bizancio, como edificios destinados al cuidado de los enfermos y discapacitados. Estos serían reintroducidos en Europa a partir del siglo XI, por el influjo de las Cruzadas y el trabajo de las órdenes religiosas y de cruzados. En su origen son un refugio para la población enferma pobre y para el aislamiento de enfermos infecciosos. Recién en el siglo XVI se les reconocería un carácter médico a los hospitales, aunque también son percibidos como un lugar para ir a morir, antes que para recuperar la salud, ya que las tasas de mortalidad hospitalaria eran escandalosas.

A lo largo del tiempo, numerosos benefactores fueron fundando hospitales: reyes, reinas, altos dignatarios eclesiásticos, nobles, comerciantes acaudalados, gremios, fraternidades y municipalidades. Todos fundaron casas para el cuidado de enfermos, pobres, débiles, ancianos y otros fines. El papel de los Gremios medievales 
también fue muy relevante en la creación de estos establecimientos medievales. Además de fundar hospitales, muchos de ellos contribuían a su financiamiento regular, como forma de brindar protección a sus miembros.

Estos hospitales medievales son instituciones de naturaleza religiosa, que perseguían un fin de cuidado espiritual. En verdad “...los hospitales no se secularizaron incluso cuando las autoridades eclesiásticas los perdieron, al hacerse cargo de ellos las municipalidadesa fines dela Edad Media. En esencia, los hospitales eran casas religiosas en las que el personal de enfermería se había reunido como una comunidad vocacional bajo una orden religiosa" (Rosen, 1984). El deseo de salvación y de santificación, como forma de evitar el dolor y sufrimiento en la próxima vida, podía lograrse a través de la realización de buenas obras, mediante la caridad con los pobres y necesitados.

Una serie de factores viene a transformarradicalmenteelfuncionamiento de esta institución medioeval. En primer lugar, desde el siglo XIII en adelante, los hospitales pasan a depender de la autoridad secular. Las autoridades municipales empiezan a hacerse cargo o a complementar las actividades de la Iglesia. Según Rosen "esta política era motivada en parte por el deseo de las autoridades civiles de hacerse independientes de la Iglesia. (...) Los monjes y las monjas continuaron dando atención de enfermería tal como lo venían haciendo. Administrativamente, las autoridades municipales eran responsables de las instalaciones hospitalarias, pero la iglesia podía tener alguna participación" (Rosen, 1984).

Además, para “...el pensamiento medieval, los pobres, los enfermos y los débiles eran considerados casi necesarios para la salvación de quien hacia una obra de caridad. (...). Pero una actitud como ésta tendía a estimular la mendicidad y la aceptación del mendigo como una necesidad. No se hacía prácticamente nada por mejorar las condiciones de los pobres y de los enfermos". El cuidado de los pobres deja de ser una preocupación cristiana, en la misma medida en que la sociedad comienza a cambiar y se ve incrementado el “ejército de reserva”(Rosen, 1984).

La Reforma y el surgimiento del Estado Absolutista impactan en este reordenamiento. "aunque la intervención de las autoridades civiles en cuestiones relacionadas con el bienestar y la salud se venía produciendo desde antes del siglo XVI, la noción de que el auxilio de los pobres, incluyendo la atención médica, era una responsabilidad de la comunidad y no de la Iglesia, sólo quedó establecida durante la Reforma”. La preocupación de estos primeros reformadores sociales tiene que ver con "eliminación de toda la mendicidad, la organización de eficientes agencias de asistencia pública, y la unificación de todas las instalaciones y recursos (hospitales, atención domiciliaria y similares en manos de autoridades municipales o nacionales" (Rosen, 1984).

Durante los siglos XVII y XVIII las políticas de población pasan a ser un tema clave. El Absolutismo y la política mercantilista generan la noción de la importancia de la salud del pueblo, para contar con una fuerza de trabajo y militar competente. Esta preocupación se fundamentaba en razones políticas, económicas y militares, como parte de un proyecto político que pone la sociedad y la economía al servicio del poder político. El Mercantilismo es una política de poder, en donde el bienestar de la sociedad es igual al bienestar del Estado. La justificación de la Política Social se encuentra en la Razón de Estado.

En Latinoamérica el esquema de desarrollo histórico es similar. Las monarquías españolas y portuguesas implantaron un modelo en el cual la Iglesia se responsabiliza del cuidado de las personas carentes de recursos. Las Leyes de Indias preveían la existencia de Hospitales en todas las ciudades del Reino, y para su administración la monarquía española 
preveía la existencia de las "Hermandades de Caridad", antiguas instituciones reales que fueron implantadas en el Reino de Indias. Disposiciones reales del siglo XVI establecían que la creación de todo nuevo asentamiento colonial debía prever la instalación del hospital para los enfermos pobres, y debían estar próximos a la Iglesia, para que la misma se encargara de su funcionamiento.

Progresivamente se produce la asociación del Hospital con la profesión médica. La idea de que el hospital debía ser tanto para el tratamiento de los enfermos como para el estudio y la enseñanza de la medicina iba a tener consecuencias extraordinariamente fructíferas en los siglos posteriores. Sobre 1700 empiezan a multiplicarse el número de establecimientos, en Londres surgen los hospitales de especialidades: 1802 Hospital para enfermedades febriles; 1804 Hospital de Oftalmología; 1814 Hospital de Tórax; 1816 de Oídos; 1838 Ortopedia.

Con el progreso de la ciencia médica, los hospitales comienzan a ser medicalizados, los criterios sociales de admisión comienzan a ser reemplazados progresivamente por criterios médicos. Los benefactores y administradores comienzan a ceder la orientación de los establecimientos a la nueva clase médica, como lugar donde se realiza la educación e investigación profesional. Con el desarrollo de la microbiología, los hospitales comienzan a ser tecnologías eficaces para recuperar la salud, y comienzan a ampliar su radio de acción. La caridad y la filantropía son totalmente insuficientes para afrontar los costos de la atención, y esto refuerza la necesidad del apoyo público estatal para el funcionamiento de los servicios hospitalarios (Healy et al., 2002)

En síntesis, la atención de enfermos y desvalidos se estructura a lo largo de la historia social. El Hospital Medieval era en esencia una institución eclesiástica, cuyos objetivos comienzan a transformarse en el siglo XVI, en un propósito social. Luego, el Hospital se secularizó, fue colocado bajo la administración gubernamental y la comunidad. Pero este hospital moderno guardó algunos rasgos medievales, porque su propósito no es puramente la atención médica, sino la preservación del orden social, gestionando socialmente a los enfermos y necesitados.

Así, los hospitales se transforman en lo que conocemos hoy: un taller de salud o industria médica. La atención médica es la preocupación principal, y su funcionamiento está guiado por normas científico-técnicas. Pero todavía guarda ciertos rasgos de su origen moderno, como hospicio para gente pobre, antes de ser centro de salud para todos. Entonces, hablar de sistemas de salud en el sentido que le asignamos actualmente, implicar relacionar este concepto con el surgimiento del Estado nacional moderno y tres fenómenos clave: la constitución de la Medicina como Profesión, el surgimiento del Hospital Moderno, y la emergencia de las formas de Aseguramiento en salud.

\subsection{La construcción política de los sistemas de salud modernos}

EneliniciodelsigloXIXeranmuypocas las personas que podían indicar que habían nacido en un Hospital. Hasta bien entrado el siglo, estas instituciones continuaron siendo parte de organizaciones de caridad, que albergaba a huérfanos, indigentes, locos, etc. Recién sobre el cierre del siglo, con el empuje de la industrialización y la visibilidad de "la cuestión obrera", se pone sobre la mesa la necesidad de organizar respuestas colectivas frente al problema de la enfermedad.

La primera preocupación se relaciona con la pérdida de ingresos que ocasiona la enfermedad, por imposibilidad de trabajar y los costos de la atención, lo cual genera respuestas en la forma de asociaciones voluntarias de ayuda mutua. Frente a ello, resulta necesario que el Estado opere como garante del orden social, regulando estos 
asuntos. En 1883 Alemania estableció el primer sistema nacional de seguro obligatorio contra enfermedad, que presta atención en salud y brinda cobertura financiera contra salarios perdidos por los obreros. Sistemas similares se establecen en Austria (1891), Noruega (1909), Inglaterra (1911), Rusia (1912) y Holanda (1913) (Starr, P. 1991).

Los países europeos instalan el seguro obligatorio de salud como parte de un programa de seguridad social, para proteger a los trabajadores de la pérdida de ingreso que ocasionaba el deterioro de la capacidad laboral. El pago por atención médica vino después. El seguro es el retorno a la protección social, pero esta vez bajo clave de ciudadanía. El descontento político, la organización de los trabajadores, la existencia de partidos socialistas, son factores explicativos en la emergencia de estos nuevos esquemas. Esto es particularmente claro en Alemania, que introdujo la primera reforma de este tipo 5 (Starr, 1991).

Los factores políticos explican la irrupción de esta innovación de los seguros sociales, en el marco de la coyuntura crítica que supuso el tránsito hacia sociedades industrializadas de masas. Pero hay factores político-institucionales que explican el sostenimiento y desarrollo posterior de estos nuevos experimentos. La existencia previa de fondos mutuos de ayuda, organizados en torno a gremios, oficios y diversas industrias, explican los diversos caminos asumidos por los Estados Nacionales en la estructuración de los seguros obligatorios.

De acuerdo a los historiadores de la salud, los Seguros Sociales de Salud (Social Health Insurance, SHI) hunden sus raíces en la historia europea, retrotrayéndose hasta la Edad Media. Los SHI actuales son

5 La elite estatal, encabezada por el Canciller Bismarck, procesa la reforma social como una forma de ganarse la lealtad política de la clase obrera, sostener el esfuerzo industrializador y construir un Estado moderno. el resultado de un proceso de experiencia acumulada en Europa Occidental en el largo plazo. Dentro de esa experiencia, podemos identificar tres corrientes de evolución institucional, asentadas y matizadas por las características nacionales.

Primero, la ampliación progresiva de la cobertura, desde esquemas que protegían a un limitado número de trabajadores en algunas ramas de actividad, hasta esquemas que abarcan a todos los residentes de un área. El esquema se desarrolla desde el bajo Medioevo, por pequeños grupos de trabajadores, Corporaciones de Artesanos, que organizan sus asociaciones de ayuda mutua. Los registros más antiguos sobre estas asociaciones datan del 1300, en países de habla alemana y sueca. El resto de la población es amparada por los cuidados que brindaban las organizaciones religiosas y de caridad (Saltman et al., 2004).

\section{Tabla 1}

Años de creación de partidos socialistas, federaciones de sindicatos y sistemas de seguridad social

\begin{tabular}{lccc}
\hline \multicolumn{1}{c}{ País } & $\begin{array}{c}\text { Partido } \\
\text { Socialista }\end{array}$ & $\begin{array}{c}\text { Federación } \\
\text { de } \\
\text { Sindicatos }\end{array}$ & $\begin{array}{c}\text { Seguridad } \\
\text { Social }\end{array}$ \\
\hline Alemania & 1875 & 1868 & 1883 \\
\hline Austria & 1888 & 1893 & 1888 \\
\hline Dinamarca & 1878 & 1898 & 1891 \\
\hline Noruega & 1887 & 1877 & 1894 \\
\hline Francia & 1905 & 1895 & 1898 \\
\hline Bélgica & 1889 & 1910 & 1900 \\
\hline Holanda & 1894 & 1905 & 1901 \\
\hline Gran & 1900 & 1868 & 1908 \\
Bretaña & 1888 & 1880 & 1911 \\
\hline Suiza & 1889 & 1898 & 1913 \\
\hline Suecia & 1892 & 1906 & 1914 \\
\hline Italia & 1890 & 1891 & 1900 \\
\hline Media- & 8,5 & 15,1 & 10,5 \\
\hline Mediana & & &
\end{tabular}

Fuente: Navarro, 1997. 
Segundo, una evolución progresiva del concepto de cobertura, desde beneficios referidos al reemplazo del salario perdido por una enfermedad o por fallecimiento, hasta el pago o provisión de servicios médicos ambulatorios, de internación y farmacéuticos. La atención en salud, su provisión directa o financiamiento, fue un desarrollo bastante posterior y hasta cierto punto secundario. Habría que esperar al gran salto científico tecnológico, y la enorme escalada de costos asociada (Starr, 1991).

Por último, el cambio en la naturaleza societaria, evolucionando desde formas autogestionadas, basada en la ayuda mutua entre trabajadores, hasta las modernas formas obligatorias legalmente, inauguradas con la reforma de Bismarck. La imbricación Estado-Sociedad se torna más densa, reforzando la dualidad entre el carácter social de la forma organizativa y el interés público de esta forma de protección social. Esta interacción estado-sociedad es característica de los primeros sistemas de protección. La ampliación de la protección social sería expresión concreta de la construcción del Estado Social, respuesta a la expansión de la ciudadanía política, que se consolidaría en la segunda mitad del siglo XX, logrando importantes niveles de cobertura poblacional y beneficios.

Los SHI tienen bases sociales fuertes, que trasuntan la alta adhesión de los ciudadanos, y explican su estabilidad frente a los profundos cambios políticos que ha vivido Europa. En términos globales, los SHI tienen tres rasgos fundamentales: son sistemas privado-sociales en cuanto a la provisión yel financiamiento; el componente de autorregulación es muy potente, los interesados gestionan directamente a través de los Fondos de Enfermedad, Asociaciones Médicas y de Pacientes; gran estabilidad financiera y organizacional.

Los SHI están diferenciados estructuralmente de los Seguros Privados lucrativos, porque forman parte de una política de ingresos, de redistribución cruzada de subsidios entre: jóvenes-viejos, sanos-enfermos, individuos-familias. El SHI es muy diferente del concepto simple de "aseguramiento", que está basado en principios actuariales precisos, como una herramienta individual para proteger el interés propio. La Solidaridad es la base del SHI, la dimensión social y psicológica, de pertenencia e identidad de grupo, de administración de valores y principios colectivos, tiene preeminencia sobre las consideraciones económicas egoístas.

Históricamente, los SHI han sido la base de construcción institucional de los sistemas modernos de protección social en salud, y todavía siguen siendo la base de los sistemas de Austria, Bélgica, Francia, Alemania, Luxemburgo, Holanda y Suiza. También es el principio de organización de varios países de Europa del Este, que debieron reconfigurar sus sistemas luego de la caída de la URSS. El SHI fue base de varios países, que sobre medios de los 705 iniciaron su transformación hacia sistemas basados en impuestos (España, Portugal, Grecia, Italia). También hay países con sistemas hoy basados en impuestos (e.g. Finlandia, Suecia, Reino Unido) que todavía tienen componentes relevantes de su financiamiento basados en cotizaciones laborales (Saltman et al., 2004).

Habría que esperar la segunda mitad del siglo XX para observar una segunda oleada de transformaciones en los sistemas de salud, inspirados en la experiencia del Servicio Nacional de Salud (National Health Service, NHS) de Reino Unido. El Reporte Beveridge había sentado las bases conceptuales de este sistema en 1942, señalando que la atención de salud es un componente clave de un sistema de seguridad social. En 1948 se instalaría el NHS como un sistema de acceso universal, en el que todas las personas, independiente de su edad, sexo, ingreso u ocupación, debían tener el derecho de acceder a servicios de salud en el momento en que lo precisaran, con servicios integrales y libres de costo, financiado con cargo a impuestos generales. 
Tabla 2

Financiamiento de sistemas de salud y año de transformación

\begin{tabular}{|c|c|c|c|}
\hline Pais & $\begin{array}{c}\text { Cotizaciones/ } \\
\text { SHI }\end{array}$ & $\begin{array}{l}\text { Impuestos/ } \\
\text { NHS }\end{array}$ & $\begin{array}{c}\text { Año de } \\
\text { transformación }\end{array}$ \\
\hline Austria & 1 & & \\
\hline Bélgica & 1 & & \\
\hline Dinamarca & & 1 & 1973 \\
\hline Finlandia * & & 1 & 1972 \\
\hline Francia & 1 & & $2000^{* *}$ \\
\hline Alemania & 1 & & \\
\hline Grecia & & 1 & 1983 \\
\hline Irlanda * & & 1 & 1970 \\
\hline Italia & & 1 & 1978 \\
\hline Luxemburgo & 1 & & \\
\hline Holanda & 1 & & \\
\hline Noruega & & 1 & 1967 \\
\hline Portugal & & 1 & 1979 \\
\hline España & & 1 & 1986 \\
\hline Suecia * & & 1 & 1970 \\
\hline Suiza *** & 1 & & \\
\hline Reino Unido & & 1 & 1946 \\
\hline Total & 7 & 10 & \\
\hline
\end{tabular}

Fuente: Saltman et al., 2004.

* En estos SHI nunca fue preponderante, el año marca el cambio desde cotización hacia financiamiento por impuestos.

** En 2002 Francia implanta impuesto a la riqueza destinado a salud.

*** Suiza implanta el seguro social obligatorio en 1996.

Este sistema de asistencia, financiada por impuestos y de cobertura universal, tenía algunos antecedentes fundamentales. Durante la década de los 2os, la URSS había logrado establecer un sistema estatal de salud. Este sistema, denominado por algunos como Modelo Shemashko, se caracterizaba por ser planificado y controlado centralmente, financiado por presupuesto del estado y sus trabajadores son funcionarios estatales ${ }^{6}$. Un sistema público, de libre acceso para todos, con servicio médico completo y gratuito.

\section{Conclusión: estructura institucional y desempeño de SHI y NHS}

Durante fines del siglo XIX $y$ principios del XX se desarrollan dos grandes modelos de protección en salud universales. Los modelos SHI, primero, apuntan a lograr cobertura de la mayoría de los ciudadanos a través de cotizaciones obligatorias a la seguridad social, de trabajadores y patronos, que tienen su origen en asociaciones voluntarias $y$ proveen servicios mediante acuerdos con efectores públicos y privados. Luego, el NHS, que centraliza el financiamiento, planeamiento y provisión en el Estado, asignando los recursos mediante un presupuesto y de acuerdo a las necesidades en salud, asegurando cobertura universal.

Los SHI están basados en acuerdos privados, pero de naturaleza pública, regulados legalmente. Su base y conducción está en organizaciones de la sociedad civil. El Estado mantiene un rol fuerte de supervisión sobre su funcionamiento. El financiamiento tiene carácter público, por la obligatoriedad de los aportes y la participación de los impuestos generales. Tiende a ser un sistema equitativo, aunque hay gente que no logra ser incorporada directamente por este esquema. Tiene un grado de aceptación y legitimidad social muy alta, pero, a su vez, tiene problemas de sustentabilidad financiera importantes, en relación a los NHS.

Los NHS, en comparación, tienen un desempeño global muy bueno, en términos de su capacidad para ofrecer servicios de calidad homogéneaparatodaunapoblación. El acceso es universal, garantizado por el financiamiento del presupuesto público.

6 Esta es la diferencia clave, porque el NHS se basa en la actuación de Médicos Generales del primer nivel de atención, que trabajan como profesionales independientes contratados. 
Tabla 3

Modelos de sistemas y opciones de reforma

\begin{tabular}{lll}
\hline \multicolumn{2}{c}{ NHS } & \multicolumn{2}{c}{ SHI } \\
\hline Fortalezas & $\begin{array}{l}\text { Amplia cobertura poblacional. } \\
\text { Baseamplia de financiamiento, basado para recaudar. } \\
\text { en impuestos. }\end{array}$ & $\begin{array}{l}\text { Menos dependencia de las } \\
\text { negociaciones políticas sobre } \\
\text { presupuesto. }\end{array}$ \\
& $\begin{array}{l}\text { Modelo degobierno sencillo, eficiencia } \\
\text { administrativa y control de costos. }\end{array}$ & $\begin{array}{l}\text { Capacidad redistributiva entre los } \\
\text { participantes. Gran apoyo social por } \\
\text { parte de usuarios. }\end{array}$ \\
\hline
\end{tabular}

Debilidades Inestabilidad presupuestaria, por Exclusión de los pobres

cambios en prioridades políticas

Fuente: Elaboración propia.

Desarrolla con éxito acciones focalizadas en grupos de población con necesidades de salud específicas. Las críticas principales se encuentran en su excesivo "burocratismo", y en su falta de flexibilidad para dar respuestas más ajustadas a las expectativas de los usuarios.

La experiencia comparativa parece concluyente, en el sentido de indicar que los NHS son superiores a los SHI en términos económicos y sanitarios. Los indicadores de desempeño en cuanto a gasto, cobertura poblacional y resultados de salud, muestran mejor desempeño de los NHS. Los SHI muestran menor capacidad para proveer servicios de salud pública: actividades de promoción, prevención, acciones intersectoriales, etc. Esto impacta en el futuro, en el marco de sociedades que profundizan sus procesos de envejecimiento poblacional. Además, la coordinación entre proveedores de servicios en el SHI es compleja, lo que dificulta la continuidad asistencial.

La Tabla 3 sintetiza las principales fortalezas y debilidades de los sistemas NHS y SHI. También presenta recomendaciones extraídos de la literatura específica, respecto a las condiciones necesarias para poder avanzar en estos modelos, como camino para la universalización de la cobertura. El punto crítico consiste en valorar adecuadamente que sistema de salud existe en los países latinoamericanos, siendo conscientes que los sistemas de protección social de nuestra región poseen una gigantesca heterogeneidad institucional 7 .

Como todos sabemos, los sistemas de salud latinoamericanos se caracterizan por la coexistencia de diferentes arreglos, donde coexisten modalidades de servicio público, seguro social y provisión privada. Las poblaciones más pobres tienden a ser atendidos en los servicios públicos de salud, hospitales y centros de salud financiados por el Estado. La población con inserción formal en el mercado de trabajo obtiene cobertura a través de la seguridad social, con marcos de cobertura variable según categoría ocupacional. Los sectores de mayores ingresos tienden a estar atendidas por seguros privados de salud o entidades de medicina integral prepagas (Terris, 1980; Londoño \& Frenk, 1997; Fleury, 20oo; CEPAL, 2006; Mesa-Lago, 2007).

Pero también hay modelos de alta cobertura, por ejemplo, en el Caribe inglés, con modelos muy homogéneos, con fuerte impronta NHS. También Cuba, que desarrolla su versión nacional del modelo

\footnotetext{
7 Sobre este aspecto, el trabajo de Midaglia y Antía (2017) muestra la diversidad de sistemas de protección social de la región.
} 
público, de inspiración Shemasko. Por otro lado, Costa Rica, con un sistema basado en seguro social de provisión pública, o Uruguay, con un seguro social de provisión de servicios público-privada. Estos casos son ejemplos de sistemas de salud que garantizan en términos generales el acceso a servicios de salud para toda su población ${ }^{8}$.

En cualquier caso, los países más rezagados en cuanto a la cobertura de sus sistemas de protección en salud muestran tres debilidades claves. En primero lugar, pobre funcionamiento del mercado de trabajo. La variable empleo, en cuanto a cantidad, calidad y formalidad, la capacidad contributiva de una sociedad, para financiar la expansión de servicios, mediante contribuciones e impuestos.

La otra variable a considerar, es la presencia de una oferta de servicios públicos de calidad. Estos no solo deben alcanzar a los sectores de menores recursos, sino también ofrecer servicios atractivos para trabajadores y clase media. En caso contrario, se corre el riesgo de que trabajadores y clases medias se direcciones hacia ofertas mercantiles, restándole su apoyo político y financiero a la expansión de los servicios públicos universales.

Otro factor crítico son las capacidades político-estatales necesarias para promover un cambio de proporciones dentro de un sistema que excluye a grandes proporciones de población. La expansión de la cobertura de salud tiene importantes consecuencias positivas en el mediano y largo plazo. Sin embargo, para los actores establecidos, que se benefician del statu quo del sistema, la apuesta siempre luce riesgosa. En esos escenarios, resulta fundamental la capacidad política del Estado, para plantear una imagen objetivo creíble, y establecer los caminos necesarios para alcanzar el

8 Brasil, con la creación del Sistema Único de Salud (SUS), creó un sistema público de orientación universalista, que al día de hoy se ve jaqueado por la insuficiencia de fondos y por el auge de los seguros privados de salud complementaria. objetivo de la cobertura universal.

En conclusión, la expansión de la protección social en salud sigue siendo un desafío importante para muchos países de Latinoamérica. La experiencia de los países desarrollados nos ofrece diversas pistas respecto a los caminos y formatos que puede asumir un sistema de protección con vocación universalista. La lección fundamental se encuentra en la importancia de la política, de las coaliciones sociales pro derechos y de las capacidades de los Estados para sostener esquemas universalistas de protección social.

\section{Referencias Bibliográficas}

CEPAL. (2006). La protección social de cara al futuro: acceso, financiamiento y solidaridad. Santiago de Chile: CEPAL.

Durán, A., Kutzin, J., Martin-Moreno, J., \& Travis, P. (2012). Understanding health systems: scope, functions and objectives. En J. Figueras, S. Lessof, M. McKee, A. Durán, N. Menabde (Ed.). Health Systems, health, wealth and societal well-being. Berkshire: Open University.

Figueras, J., Lessof, S., McKee, M., Durán, A., Menabde, N., (2010). Health Systems, health, wealth and societal well-being. Berkshire: Open University.

Figueras, J. \& McKee, M. (2012). Health systems, health, wealth and societal well-being: assessing the case for investing in health systems. New York: Open University.

Fleury, S. (200o). Remodelando los servicios de salud en América Latina. Un análisis comparativo de la reforma en México, Argentina y Brasil. Otawa: IRDC-CIID.

Freidson, E. (1970). La profesión médica. Madrid: Ediciones Península.

Healy, J. \& Mckee, M. (2002). Hospitals in a changing Europe. Philadelphia, USA: Open University.

Londoño, J. L. \& Frenk, J. (1997). Pluralismo estructurado: hacia un modelo innovador para la reforma de los 
sistemas de salud en América Latina. Recuperado de http://repository. unad.edu.co/bitstream/10596/4826/1/ pubwp-353\%20(1).pdf

Marset Campos, P. \& Saez Gómez, J. (1997). La evolución histórica de la salud pública. Madrid.

Mesa-Lago, C. (2007). Reassembling social security. A survey of pensions and healthcare reforms in Latin America. New York: Oxford Press.

Midaglia, C., Antía, F. (2017). Bienestar y protección social en América Latina: Abordajes conceptuales y metodológicos para el MERCOSUR. Asunción: ISM.

Mladovsky, P., Srivastava, D., Cylus, J., Karanikolos, M., Evetovits, T., Thomson, S., McKee, M. (2012). Health policy responses to the financial crisis in Europe. Copenhague: Open University.

Navarro, V. (1997). Salud pública. Madrid: McGraw Hill Interamericana.

OMS. (2010). Informe sobre la salud en el mundo: la financiación de los sistemas de salud: el camino hacia la cobertura universal. Ginebra.

RAE. (2017). Salud. En Diccionario de la Lengua Española de la Real Academia. Recuperado de http://dle.rae. es/?id=X7MRZku

Roemer, M. I. (1985). National strategies for health care organization. A world overview. Michigan: Health Administration Press.
Rosen, G. (1984). De la policía médica a la medicina social. Ensayos sobre la historia de la atención a la salud. México: Siglo XXI Editores.

Saltman, R., Allin, S., Mossialos, E., Wismar, M. \& Kutzin, J. (2004). Assessing health reform trends in Europe. Copenhague: WHO.

Setaro, M. (2013). La economía política de la reforma del sector salud en Uruguay (Tesis doctorral). UDELAR. Montevideo, Uruguay.

Siegrist, J. (2000). The ocial causation of Health and Illness. En G. Albrecht, R. Fitzpatrick \& S. Scrimshaw (Ed.). Handbook of Social Studies in Health and Medicine. sl: SAGE.

Starr, P. (1991). La transformación social de la medicina en los Estados Unidos de América. México: Biblioteca de la Salud. Fondo de Cultura Económica.

Terris, M. (1980). Tres sistemas mundiales de atención médica. Cuadernos Médicos Sociales, (14).

Villar, H. (2003). La salud: una política de Estado. Hacia un sistema nacional de salud. Marco conceptual, el contexto, situación actual y propuestas de cambio. Montevidel: Imprenta Grafinel.

Witheford, M. \& Lacivita, L. (2000). Comparative Health Systems: emerging convergences and globalization. En: Albrecht, A., Fitzpatrick, R. \& Scrimshaw, S. Handbook of Social Studies in Health and Medicine. SAGE. 